\title{
AMENAZAS Y OPORTUNIDADES DE LA ECONOMÍA DIGITAL EN EL MERCADO LABORAL DE MÉXICO*
}

\author{
PATRICIA CARMINA INZUNZA-MEJÍA***
}

Fecha de recibido: 26 de junio de 2017

Fecha de aprobado: 30 de mayo de 2018

\begin{abstract}
Resumen
En este artículo de revisión de literatura, se tiene por objetivo analizar la perspectiva que tienen los trabajadores en México ante las amenazas y oportunidades que ofrecen las tecnologías de la información y de la comunicación (TIC) en el mercado laboral, lo que implica revisar el basamento teórico como algunos referentes empíricos institucionales que revelan una realidad contraída, pero que también aportan reflexiones positivas acerca de las bondades de la economía digital para empleados y empleadores. Entre los hallazgos, se reconoce que, si los cambios tecnológicos desplazan a los trabajadores, al sustituir al hombre por máquinas tecnológicas, también este proceso de desplazamiento genera alternativas de empleo en aquellos sectores de la economía con capacidad de absorber los trabajos desplazados, sobre todo si se trata de actividades no automatizables. Además, con el uso de las TIC, se advierten oportunidades de empleo en el mercado laboral y, en consecuencia, tanto empleadores como trabajadores deberán ser resilientes digitales y adaptarse a los cambios que impone la economía digital, para lo cual se deberán formar en habilidades y competencias tecnológicas y digitales.
\end{abstract}

Palabras clave: economía digital; mercado laboral; resiliencia digital; habilidades digitales; competencias tecnológicas.

\section{THREATS AND OPPORTUNITIES OF THE LABOR MARKET IN THE DIGITAL ECONOMY OF MEXICO}

\begin{abstract}
This literature review article aims to analyze the perspective of workers in Mexico on the threats posed and opportunities offered by information and communications technology (ICT) in the labor market. This implies reviewing the theoretical basis and some institutional empirical references that reveal a contracted reality, but also contribute positive reflections on the benefits of digital economy for employees and employers. The findings suggest that, if technological changes displace workers by replacing man with technological machines, such displacement will also create alternative employment in those sectors of the economy that are able to absorb displaced jobs, especially non-automatable activities. In addition, ICT will possibly provide job opportunities and, consequently, both employers and workers shall be digitally resilient and adapt to the changes imposed by digital economy, for which they shall develop technological and digital skills.
\end{abstract}

Keywords: digital economy; digital resilience; digital skills; labor market; technological skills.

* Artículo de reflexión.

** Profesora e investigadora de Tiempo Completo, Titular "C" de la Universidad Autónoma de Sinaloa (México). Magíster en Negocios y Alta Dirección, doctora en Estudios Fiscales. Correo electrónico: inzunzap@uas.udu.mx 


\title{
AMEAÇAS E OPORTUNIDADES DA ECONOMIA DIGITAL NO MERCADO DE TRABALHO NO MÉXICO
}

\section{Resumo}

\begin{abstract}
Este artigo de revisão de literatura tem por objetivo analisar a perspectiva que os trabalhadores no México têm ante as ameaças e oportunidades que as tecnologias da informação e comunicação (TIC) oferecem no mercado de trabalho, o que implica revisar referenciais teóricos empíricos institucionais que revelam uma realidade contraída, mas que também contribuem com reflexões positivas sobre os benefícios da economia digital para empregados e empregadores. Entre os achados, reconhece-se que, se as mudanças tecnológicas deslocam os trabalhadores ao substituir o homem por máquinas tecnológicas, esse processo de deslocamento também gera alternativas de emprego em setores da economia com capacidade de absorver os trabalhos deslocados, principalmente se se tratar de atividades não automatizadas. Além disso, com o uso das TIC, evidenciam-se oportunidades de emprego no mercado de trabalho $e$, em consequência, tanto empregadores quanto empregados deverão ser resilientes digitais e adaptar-se às mudanças que a economia digital impõe; para isso, deverão ser formados em habilidades e competências tecnológicas e digitais.

Palavras-chave: economia digital; mercado de trabalho; resiliência digital; habilidades digitais; competências tecnológicas.

Inzunza-Mejía, P. C. (2018). Amenazas y oportunidades de la economía digital en el mercado laboral de México. Revista de la Facultad de Ciencias Económica: Investigación y Reflexión, XXVI(2), DOI: https://doi.org/10.18359/rfce.2926
\end{abstract}

JEL: J24, J49, O54.

\section{Introducción}

La economía digital está avanzando en muchas direcciones e influyendo en la forma como vivimos, lo que provoca la apertura de nuevas industrias y nuevos modelos de negocio, aunado a los cambios en la forma de organizar el mercado laboral. El uso desarrollado de las tecnologías de la información y de la comunicación (TIC) y el gran impacto de internet en todos los sectores de la economía las caracteriza como tecnologías de uso general, con el potencial de transformar la economía y aumentar la productividad. Por esto, el estudio se limita al análisis de la transformación digital del mercado laboral como un sector permeado por la economía digital.

En la medida en que el uso y acceso a internet se incrementa en México y el mundo, los instrumentos tecnológicos de conectividad y los grandes flujos de información se convierten en catalizadores cada vez más importantes del crecimiento económico. Sin embargo, se podrian aprovechar las oportunidades de empleo que generan los cambios tecnológicos, pero dependerá de la capacidad del sector público, los empresarios y la sociedad para invertir y para adaptarse a los cambios que las TIC imponen.

En este contexto, el objetivo del presente artículo es analizar las amenazas y oportunidades que enfrenta el mercado laboral en la economía digital para explicar los efectos de las tecnologías sobre los trabajadores y el empleo, y analizar las acciones de política pública necesarias que permitan facilitar el tránsito de la economía tradicional a la economía digital, destacando el caso del mercado laboral mexicano.

\section{Metodología: materiales y métodos}

Según las argumentaciones teóricas y empíricas analizadas, la metodología cualitativa que se aplicó para este estudio es descriptiva y explicativa, bajo 
el modelo de investigación científica modular por objeto de transformación, desarrollado mediante el análisis documental de referentes teóricos y otros estudios empíricos que reflejan la realidad que corresponde en general a países desarrollados y en particular a México.

El estudio del mercado laboral en la economía digital surge ante la preocupación del desplazamiento de mano de obra por el uso de las TIC, y también para reconocer de manera objetiva que dicho uso y los ecosistemas digitales que encierra son un área de oportunidad para el mercado laboral en general. Bajo esta idea, con la revisión bibliográfica, se recopila la información más notable sobre este tema con el propósito de identificar lo que se conoce de este, qué se ha investigado y qué aspectos permanecen desconocidos, a fin de dar respuesta a las preguntas planteadas acerca de ¿qué perspectiva laboral tienen los trabajadores ante la economía digital? y ¿las TIC están desplazando la fuerza laboral tradicional?

En la primera etapa de la metodología, se realiza una revisión de literatura científica que permite identificar procesos, determinantes y procedimientos implicados en el desplazamiento laboral por el uso de las TIC como una de las principales amenazas para los trabajadores.

Para la localización de los documentos científicos, en febrero de 2017, se realizó una búsqueda bibliográfica en diversos buscadores científicos en internet, entre ellos, Scopus, Redalyc y Dialnet, utilizando descriptores y operadores boleanos. Se procedió a la descripción de las principales respuestas mediante lectura crítica y escritura científica, revisión de artículos científicos, construcción de fichas bibliográficas y notas claves, y realización de mapas conceptuales.

Tras la combinación de palabras claves, se registró un acervo de 84 artículos que trataron el tema en los últimos cinco años, posteriormente se limitó la búsqueda a solo países latinoamericanos, reduciendo la selección de artículos a un $20 \%$, de los cuales solo el $5 \%$ corresponderían a México. Esto nos demostró que en el país existe muy poca literatura científica que desarrolle estos temas.
La etapa de revisión de artículos científicos, lectura crítica y escritura consistió en el siguiente procedimiento:

- Resumir información sobre el tema o problemas.

- Se identificaron los aspectos relevantes que dieran respuestas a las preguntas de investigación.

- Se analizaron aspectos desconocidos y los más desarrollados por los autores.

- Se describieron las aproximaciones teóricas y empíricas eslabonadas.

- Se identificaron aproximaciones metodológicas al estudio.

- Se desarrollaron fichas bibliográficas que precisen las variables asociadas al estudio del tema.

- Se registra información amplia sobre un tema, tanto de documentos institucionales como de documentos científicos.

- Se procedió a documentar la discusión de manera crítica que permitiera el desarrollo de conclusiones de ideas contradictorias e ideas coincidentes procedentes de diferentes estudios.

- Se repite la lectura de documentos primarios.

- Se preparó la comunicación reflejada en este trabajo.

- Se procuró un medio de difusión para contribuir a superar las barreras de producción y difusión del conocimiento.

- Se procedió a atender con interés y cuidado la discusión crítica del comité evaluador del medio que divulga este artículo.

- Se prepararon los cambios para mostrar la evidencia disponible.

- Se dio respuesta a las preguntas planteadas y se generaron nuevas preguntas. 
- Se genera presencia del tema y sugieren aspectos o temas de investigación.

Para el análisis de los artículos científicos e institucionales, se utilizaron las técnicas descriptivas y narrativas, que consistieron en una revisión clásica de literatura. Con el enfoque cualitativo, tras seleccionar un determinado número de artículos, se agruparon en general por el sentido de sus resultados que se discutieron a la luz de las características metodológicas de cada estudio, para producir una aseveración narrativa sustentada en estudios previos que permitieran concluir y dar respuestas a las preguntas planteadas.

En la descripción temática, se brida al lector la oportunidad de ponerse al día sobre evidencia empírica, cotidiana y conceptual en áreas en constante evolución, como la economía digital o el desplazamiento laboral por el uso de las TIC. Este tipo de modalidad descriptiva es pertinente en la enseñanza de la economía digital y también es de interés de muchas personas de campos conexos como el mercado laboral. Leer temáticas tan actuales como la economía digital y su incidencia en el mercado laboral permite visibilizar el escenario que les espera tanto a trabajadores como a empleadores, lo que hace posible una esfera general de interés e incidencia de intereses del mercado laboral.

\section{Problemas y contradicciones de la economía digital en el mercado laboral}

Con el estudio teórico, además de que se impulsa un espacio de análisis, discusión y diálogo para la reorientación de la investigación, se procede al desarrollo de la estrategia metodológica a partir de las principales definiciones.

En tal sentido, se define la economía digital como la economía de internet, y según Nottebohm, Manyika, Bughin, Chui y Syed (2012), corresponde a las actividades asociadas a la creación y el uso de las redes y de los servicios de internet, incluso la totalidad de las actividades de internet y la parte del sector de las TIC que están relacionadas con la web. Se consideran cuatro categorías: a) actividades que utilizan la web como soporte (comercio electrónico, creación y distribución de contenido y publicidad online), b) telecomunicaciones sobre protocolo de internet (IP) o relacionadas con comunicaciones IP (proveedores de servicios de internet [ISP]), c) software y servicios facilitados por internet (consultoría de TIC y desarrollo de software) y d) manufactura de hardware y proveedores de mantenimiento de equipos (computadoras, teléfonos inteligentes, equipo de hardware y servidores).

Un informe reciente de la Organización Internacional del Trabajo (OIT) y de la Comisión Económica para América Latina y el Caribe (Cepal, 2013) nos advierte sobre una crisis para los mercados laborales en la economía digital para el periodo de 2016 a 2017, lo que nos obliga a reflexionar sobre ¿cómo vamos en el mercado laboral? y ¿qué tan competitivos somos? Esto significa que el mercado laboral es un eslabón dentro de la cadena de valor laboral de la economía digital.

Por un lado, Frey y Osborne (2017) sostienen que el uso generalizado y en aumento de las TIC conlleva sucesos de pérdida masiva de puestos de trabajo, dado que, no solo se automatizan las tareas rutinarias del trabajo, sino que también se exponen al riesgo las tareas cognitivas que hasta hace poco se consideraban no automatizables. Algunas de las estimaciones más pesimistas sugieren que cerca de la mitad de los empleos en los Estados Unidos están en riesgo de ser sustituidos por máquinas dentro de los próximos diez a veinte años.

Estos planteamientos remiten a los estudios de Acemoglu y Restrepo (2016), quienes creen que la dinámica de los mercados laborales modernos se caracteriza por la trayectoria entre dos fuerzas estimuladas por la tecnología: a) la automatización, del lado de las máquinas, y b) la creación de nuevas funciones complejas para los humanos. Ambas fuerzas han estado equilibradas. Para explicar este hecho, Acemoglu y Restrepo sostienen que la automatización y la creación de nuevas funciones no son independientes entre sí, dado que la automatización de los empleos existentes reduce la demanda de empleo y tiende a reducir los costes laborales, pero también hace que la creación de nuevas funciones complejas para los seres humanos sea más rentable en relación 
con una mayor automatización. Este efecto precio podría actuar como una fuerza de estabilización que, con el tiempo, tiende a autocorregir la pérdida de empleos provocada por la tecnología.

Po otro lado, Arntz, Gregory y Zierahn (2016) consideran que para 21 países desarrollados de la Organización para la Cooperación y el Desarrollo Económicos (OCDE), en promedio, solo el $9 \%$ de los empleos están en alto riesgo de ser automatizados. Por su parte, encuentran que una proporción mayor de ocupaciones tiene bajo riesgo de ser totalmente automatizadas, pero consideran que entre un 50 y un $70 \%$ de tareas laborales podrían ser reemplazadas o transformadas radicalmente.

Sin embargo, un estudio del Banco Mundial (2016, p. 14) expone que

el número de empleos directos que crean las tecnologías digitales es bastante pequeño, pero la cantidad de puestos de trabajo que habilitan puede ser importante. En los países en desarrollo, el sector de las TIC representa, en promedio, apenas alrededor del $1 \%$ de los trabajadores, mientras que en los países miembros de la OCDE, entre el $3 \%$ y el $5 \%$ del empleo corresponde a este sector.

Según este contexto, se observa que el mayor potencial de generación de empleo no está en el sector de las TIC, pues el número de empleos directos que este genera es muy pequeño.

Bajo estas evidencias, recurrimos a los argumentos de Acemoglu y Restrepo (2017), al expresar que la automatización tiene varios efectos en el mercado laboral. Por una parte, las TIC pueden desplazar a trabajadores que desempeñan determinados trabajos en ciertas industrias, lo que disminuye sus oportunidades de empleo y sus salarios. Por otro lado, hacen hincapié en que, a medida que los robots y otras tecnologías asistidas asumen las tareas previamente realizadas por el trabajo, hay una creciente preocupación por el futuro de los empleos y los salarios, y analizan el efecto del aumento en el uso de robots industriales entre 1990 y 2007 en el mercado laboral. Utilizando un modelo en el que los robots compiten contra el trabajo humano en las diferentes tareas y muestran que ellos pueden reducir el empleo y los salarios, y que sus efectos en el mercado laboral se pueden estimar mediante la regresión del cambio en el empleo y en los salarios sobre la exposición a los robots en cada mercado de trabajo local.

El impacto de los robots se da en la disminución de los empleos de rutina, la deslocalización, otros tipos del stock total de capital. Según sus estimaciones, un robot por cada 1000 trabajadores reduce el empleo a población entre un 18 y un $34 \%$ y en los salarios en un 25 y un $50 \%$. Sin embargo, otros sectores y ocupaciones pueden expandirse y absorber la mano de obra liberada de las tareas que realizan las máquinas, e incluso es factible que las ganancias en productividad empujadas por nuevas tecnologías amplíen las posibilidades de empleo en las industrias en las que se despliegan.

\section{Discusión de la literatura: mercado laboral como un eslabón de competitividad}

La competitividad se define como la capacidad que tiene una unidad económica o un país para competir en los mercados de bienes y servicios (incluido el mercado laboral).

Desde esta perspectiva, las organizaciones como empleadores deben cumplir los planes estratégicos de competitividad con cimientos tecnológicos y, en consecuencia, los trabajadores deberán apoyarse en herramientas tecnológicas para desarrollar tanto procesos administrativos como procesos de manufactura, distribución y compraventa, con la finalidad de que el entorno laboral se adapte a los cambios que el ecosistema digital establece. No obstante, si los empleadores no adaptan sus procesos al uso de las TIC, se corre un alto riesgo de perder el eslabón de competitividad y, con ello, ofrecer un escenario laboral arcaico y rudimentario en los procesos, lo que desincentiva competencias laborales orientadas a las TIC. 
La capacidad y las competencias en las TIC deben desarrollarse tanto en empleados como en empleadores. Adaptarse a las tecnologías cambiantes implica que los trabajadores se inclinen a la resiliencia laboral digital que impacta la estructura del mercado laboral. Esta capacidad de resiliencia laboral digital, de acuerdo con Dahms (2010) y Diodato y Weterings (2015, p. 724), representa "la capacidad de un trabajador para responder rápidamente a la inseguridad y los riesgos que implica adoptar las TIC en tareas laborales (la traducción es mía)"; es decir, es la capacidad adaptativa de las estructuras tecnológicas al trabajo en particular y al mercado laboral que debe adaptarse a los cambios.

En Duffi (2016, p. 441), se identifica que por parte de los trabajadores existe un gran interés en las tecnologías cambiantes que impactan el trabajo creativo, dado que gran parte de los recientes cambios en la producción y en los servicios inciden positivamente en el posicionamiento del trabajo digital. Como referencia, ha sido destacable la producción digital en el campo de la moda, la belleza, los servicios y el comercio al por menor, que ha impactado la forma en que se articulan y se da valor a las actividades económicas que podrían ofrecer un puesto de trabajo.

Un estudio del Centro de Estudios de las Finanzas Públicas (CEFP, 2017, p. 4) reveló que la competitividad basada en procesos tecnologizados permite comparar la estructura de costos del proceso de producción, como mano de obra, materias primas, tecnología, diferenciación de productos y tamaño del mercado, de un productor con respecto a otros productores de bienes internos o externos con niveles de calidad similares. Para el CEFP, entre los principales factores que inciden en la medición de la competitividad del mercado laboral basada en las TIC, se encuentran los costos laborales, las regulaciones laborales, la calidad de la mano de obra, las estrategias comerciales con uso de las TIC (mercadotecnia e innovación) que integran cadenas de valor y el progreso tecnológico en el escenario laboral de cualquier actividad económica.

En los últimos años, México ha transitado por cambios estructurales, varios de ellos sustentados en las recientes reformas estructurales que han tenido lugar a partir de la década de los ochenta. Las últimas de ellas aprobadas por el Congreso en el periodo de 2013 a 2014, que, si bien algunas aún no muestran resultados contundentes y de corto y largo plazo sobre las condiciones del mercado laboral, sí están fundamentando nuevas estructuras de costos, negocios y conglomerados, principalmente en los nichos laborales de las industrias manufactureras y del sector energético (CEFP, 2017, p. 5).

Según esto, se observa que los trabajadores mexicanos se enfrentan a un contexto poco competitivo, en el que tienen escaso acceso a las TIC y en el que cada vez les alcanza menos su salario para adquirir la canasta básica, de ahí que "los salarios necesitan tener una base más alta para que el rebote sea una base más alta y que no tengan que tocar fondo para comenzar a subir", por lo que propone el proyecto Impacto del cambio tecnológico en el mercado de trabajo de México, con el objetivo de mostrar los cambios en las tasas de participación económica y de inserción sectorial y ocupacional de la mano de obra digital de México.

\section{Oportunidades de empleo y competitividad del mercado laboral}

Desde la perspectiva de la economía digital y de su ecosistema, se prevé el surgimiento de nuevos empleos como científico de datos, traductores, manejadores de big data, agentes en ciberseguridad y suscriptores de seguros $\mathrm{O}$, incluso, actividades relacionados con los diagnósticos médicos, distribuidor de productos adquiridos vía internet, o desarrollador web, entre otros. Según el Banco Mundial (BM, 2016), se distingue que las mayores oportunidades para elevar el empleo se podrían observar en los sectores de la economía que adopten satisfactoriamente las TIC o que simplemente las aprovechen para hacer crecer la mano de obra que se desplaza a actividades aptas de ser automatizadas a través del uso de estas.

Según el BM (2016), los riesgos de desplazamiento laboral de las TIC existen, aunque no son tan elevados, dado que un empleo en la industria de alta 
tecnología en países desarrollados generaría un promedio de tres a cinco empleos adicionales en otros sectores de la economía, lo que crearía un efecto de rebote positivo y compensatorio en el mercado laboral de otros sectores económicos.

A diferencia de las transformaciones tecnológicas del pasado, como la mecanización de la agricultura o la automatización de las manufacturas, las TIC e internet afectan más a los empleos administrativos destinados a los servicios y al comercio al por menor (BM, 2016, p. 22), con lo cual se encuentra que los empleos administrativos, de servicios y de atención al cliente serían los de más alto riesgo de ser desplazados por el uso de las TIC.

Los avances tecnológicos establecen nuevas formas y actividades laborales, dado que introducen tareas novedosas y más complejas para las que en general los humanos tienen (al menos inicialmente) una ventaja comparativa. Estas tareas originan nuevos trabajos, como programadores de aplicaciones informáticas, gestores de marketing digital, expertos en ciberseguridad, científicos de datos o abogados especializados en privacidad digital. Por su parte, Acemoglu y Restrepo (2016) documentan la importancia de las nuevas tareas en el crecimiento del empleo a partir de datos del mercado laboral estadounidense. Sostienen que casi la mitad del crecimiento total del empleo desde 1980 a 2007 (el $8,8 \%$ del $17,5 \%$ ) se explica por el aumento adicional en aquellas ocupaciones con nuevos títulos de trabajo, en los que los trabajadores desempeñan tareas tecnologizadas más novedosas que aquellas de los puestos tradicionales y rudimentarios.

Los nuevos trabajos creados por sectores tecnológicos aumentan la demanda de servicios en la economía local y, por tanto, generan puestos de trabajo indirectos. Este efecto multiplicador es mayor para los empleos cualificados, como es el caso de los sectores tecnológicos. Goos, Konings y Vandeweyer (2015) estiman que para Europa cada empleo del sector de alta tecnología (que incluye tanto la fabricación como los servicios intensivos en conocimiento) genera cinco puestos de trabajo adicionales de baja tecnología en la región en la que se ubica la industria. La misma cifra considera Moretti (2010) para el sector de alta tecnología en los Estados Unidos. Estas cifras, junto con el hecho de que los avances tecnológicos producen oportunidades de empleos cada vez más cualificados e indican que el tamaño del multiplicador local desempeñará un papel esencial en el futuro del empleo y del mercado laboral a nivel mundial.

Por su parte, Duffi (2016) destaca otro aspecto de los discursos de la autenticidad, la construcción de la comunidad fashion y la dedicación-afinación de la marca, con características de representación de género pero con miras al futuro digital y empresarial de la creatividad y la tecnología. Lo llama "trabajo digital aspiracional", por generar oportunidades a los trabajadores que aspiran a realizar actividades productivas desde un ambiente digital, sin descuidar la promesa de capital social y económico. No obstante, el sistema de recompensas para estos aspirantes es muy desigual.

De hecho, el trabajo digital genera que unos pocos elegidos pueden alcanzar sus objetivos profesionales a través de las TIC, pero esta ideología del trabajador ensombrece las construcciones de rentabilidad y resalta los problemas de subjetividades de desarrollo profesional, éxito laboral, género y de clase, que bien podrían abordarse en investigaciones futuras.

En este contexto, con el uso de las tecnológicas en el trabajo, habrá cada vez más oportunidades para los trabajadores que se emplean en organizaciones que ofrecen bienes y servicios por internet (marketplaces, big data, advertising technology y software as a service, cowd computing, entre otros). También, es un hecho que hay inversionistas nacionales y extranjeros que buscan organizaciones destacadas en el país que ofrezcan servicios de este tipo.

\section{Búsqueda de empleo, autoempleo y comunidades digitales}

Un porcentaje muy bajo de los puestos de trabajo se consiguen a través de la búsqueda de trabajo en portales web, e incluso algunas ofertas de trabajo no aparecen en los canales habituales; por ello, la búsqueda de empleo se debe abordar de manera 
continuada y con dedicación, ya que la búsqueda de trabajo es en sí un trabajo.

Es sabido que para encontrar trabajo las personas se tienen que vender competitivamente en capacidades y competencias en las TIC, posicionarse laboralmente al trabajar con disciplina y compromiso, hacerse querer y destacar sobre el resto de sus compañeros de trabajo. En esa multitud de personas que conforman el mercado laboral, la información desempeña un papel importante, ya que hace que los canales tradicionales de búsqueda ya no sean suficientes para conseguir un empleo en los sitios web, las redes sociales, las notas de consultoras de selección laboral, y otros medios virtuales.

Sin embargo, Ruberg (2016, p. 147) expone que los estudios de trabajos digitales han sido insuficientes y muchos de los pocos que existen se orientan a dar atención al trabajo sexual en línea, lo que constituye una parte importante del mercado de trabajo basada en la web contemporánea. Existe suficiente evidencia del aumento de la pornografía aficionada de estilo tubo, que circula a través de los sitios web como YouTube.

Esta tendencia apunta hacia un cambio importante en la forma en que el contenido para adultos está siendo producido y distribuido en espacios digitales, pero, sobre todo, en la manera de desarrollar un trabajo; de ahí que Ruberg (2016, p. 149) explora la política de mano de obra que se basa en el trabajo remunerado de hágalo usted mismo (do-it-yourself) y reciba su pago vía internet. Esta modalidad de uso de las TIC para el trabajo permite al segmento de la industria porno poblar y posicionar sitios del tubo altamente lucrativos con cientos de miles de videos de aficionados colgados digitalmente.

Así es que de manera alternativa se da reconocimiento al sector digital mediante la producción de pornografía bricolaje como mano de obra digital que ofrece la oportunidad de desafiar los esquemas laborales tradicionales y hacen que la red de las fuerzas capitalistas que impulsan el contenido en línea de aficionados libres sea más visible. Esto también presenta un valioso marco a través del cual criticar la idea errónea de que el trabajo digital podría ser una panacea, sobre todo por tratarse de cuestiones sexuales dañinas.

Esto hace que se reflexione acerca de los medios que se tienen para solicitar y desarrollar empleo, dado que las personas no tienen que dirigirse únicamente a los sitios web o redes sociales más comunes de ofertas de empleo; al contrario, deben enfocar su búsqueda en todas direcciones, incluso tanto en los medios digitales como en los tradicionales o físicos: boca a boca (el empleo puede estar en el vecino, amigo o pariente más cercano), aun cuando la población que se encuentra en búsqueda activa de empleo tiene que hacerse ver en internet para poder descartar sus habilidades y competencias en las TIC.

El individuo tiene que manifestar sus conocimientos, su capacidad y compromiso, que a su vez definen en él una solvencia profesional y laboral. Una opción es la creación o participación en blogs relacionados con la actividad por desarrollar en el futuro puesto de trabajo, y otra es interactuar de manera continua en cualquier lugar relacionado con la actividad laboral.

En este sentido, no se habla únicamente de personal cualificado en el ámbito de la tecnología, sino de cualquier empleo: obreros, electricistas, cocineros, desarrolladores web, directivos de ventas, contadores, diseñadores, estilistas, entre otros. Por ejemplo, a través de las TIC, un estilista puede identificar los foros de mujeres o marcas de cosméticos, conocer la información que cualifica su mercado y contestar comentarios o dudas que tenga la gente sobre este ámbito. Esa iniciativa deriva en construir una imagen de confianza, proporcionándole referencias y finalmente oportunidades de trabajo en un ambiente de la economía digital.

Una vez conseguido el puesto de trabajo, no se debe abandonar la idea de imagen digital, sino que se ha de hacer un hábito que forme parte de la personalidad del servicio que se ofrece o del ADN del trabajo que se proporciona, ya que para futuros empleos será imprescindible el uso de las TIC en la economía. La digitalización de productos y procesos no se debe dejar solo para cuando se busca empleo. 
La importancia de la automatización del trabajo manual, de la descentralización de los centros de mando y decisión, y de la agilidad en la gestión y el uso intensivo de las TIC son algunas de las características de este nuevo tipo de ecosistemas digitales en ambientes laborales (Tabarés, 2016).

En los últimos años, se ha caído en el error o en la indolencia de enfocar todos los esfuerzos solo en la web, descuidando el networking, el cual puede aportar credibilidad e incluso oportunidades con la persona que se desea trabajar; de ahí la importancia que tiene "trabajar" mientras se busque el empleo ideal. Hay variados sitios en internet donde se puede acceder a un mercado laboral digital, para tener trabajos de tiempo parcial, mantenerse actualizado y activo.

Para Campos y Alonso (2015), la oportunidad laboral puede estar donde menos se espera, por lo que se plantean exponer las herramientas digitales en línea para la autopromoción laboral, tras lo cual concluyeron que la red social es el mejor componente del e-Recruitment, que es la utilización de las herramientas sociales en línea ante el reclutamiento de nuevos empleados y su relación con empleadores. Mientras que para López y Saladrigas (2016) es importante analizar el efecto y el uso de las TIC en la construcción del desarrollo local y el impacto del trabajo de los jóvenes y su empoderamientos social.

Para la búsqueda de empleo, autoempleo y comunidades digitales, es necesaria la democratización digital. Kulesz (2016) plantea que la misión de democratizar la prestación de servicios públicos de telecomunicaciones y de las TIC, incluso la radiodifusión y la televisión, significarían un área de oportunidad para el mercado laboral, por lo que se induciría la consolidación de la infraestructura de banda ancha, fundamentalmente entre los sectores menos atendidos en México, conectados al plan de suministro de banda ancha en espacios públicos.

Con este fin, Kulesz (2016) explica que el Programa Sectorial de Comunicaciones y Transportes se enfoca en optimizar la cobertura, conectividad y accesibilidad de las comunicaciones en el ámbito de la televisión, de la radio, de la telefonía y de los datos, lo que permitiría una mayor cobertura de las TIC a posibles personas que buscan empleo.

Desde ese punto de vista, López y Saladrigas (2016) mencionan que es importante el uso de las TIC en la sociedad actual. Examinan las potencialidades que ellas pueden tener en el mercado laboral, el desarrollo social y comunitario de las localidades, sin perder la perspectiva de los entornos particulares y diferenciales que utilizan iguales o similares tecnologías que alcanzan niveles de desarrollo social diferentes en las comunidades digitales.

La búsqueda de empleo y autoempleo en las comunidades digitales se corresponde al sector de la infocomunicación. Hace aproximadamente dos años Campos y Alonso (2015) destacaron que dentro de las comunidades digitales el sector de las infocomunicaciones es donde internet ha alcanzado mayor penetración como medio de difusión, superando el $90 \%$ de las empresas encuestadas. En un segundo lugar, y a gran distancia, se encuentra la industria $(69,11 \%)$, la construcción $(68,73 \%)$ y el sector de servicios (67,06 \%). Los contactos personales se muestran como la principal fuente de búsqueda de trabajo por parte de los candidatos $(97,2$ $\%)$, seguido de la búsqueda en portales de empleo $(76,42 \%)$ y la consulta en la web de las empresas (73,15\%). Finalmente, para la búsqueda de empleo en el extranjero, son los portales de empleo nacionales (43,2 \%), los buscadores de internet (38\%), los portales de empleo extranjeros $(36,1 \%)$ y las páginas web corporativas ( $32 \%$ ) los más usados por los candidatos.

Para López y Saladrigas (2016), lo novedoso es que esta forma de comunicación de las comunidades digitales aparece generada por la convergencia de las telecomunicaciones, la informática y la industria de contenidos en una sola plataforma tecnológica que permite que las experiencias comunicacionales y los procesos de interrelación alcancen una difusión y se multipliquen en la sociedad globalizada, gracias a los sistemas de transmisión, como los satélites o la fibra óptica, pero, sobre todo, a la posibilidad de transmitir mensajes digitales de todo tipo (textos, imágenes, sonidos, videos) por la red. En resumen, es posible que la comunicación personal o local conquiste es- 
pacios globales y que las experiencias de comunicación de pequeñas comunidades se socialicen a gran escala gracias a los medios digitales.

Por esto, López y Saladrigas (2016) sostienen que la infocomunicación en la sociedad es un proceso que se sustenta en un conjunto de programas que ejecutan proyectos dirigidos a desarrollar armónicamente una infraestructura técnica determinada por artefactos físicos, hardware y software, sistemas de información, telecomunicaciones y aplicaciones que faciliten el uso de servicios remotos a los trabajadores entrenados o preparados.

A manera de reflexión final, comprendemos que las TIC en la sociedad en general y en el mercado laboral en particular han contribuido a generar $y$ construir el desarrollo económico y laboral de comunidades y regiones.

\section{Resultados: el mercado laboral en la economía digital}

Para finalizar esta primera aproximación teórica acerca del mercado laboral en la economía digital, se recurre a los planteamientos de Castillo y Lechuga (2005, p. 63), quienes sostienen que países como México deben tomar las ventajas de aplicar las TIC de los países avanzados y no tratar de inventarlas; pese a que México inicie con mucho menos capital por trabajador, por lo que su desarrollo tiene un amplio campo para un rápido crecimiento comprando tecnología y copiando los métodos de producción de los países desarrollados, lo que le permitiría crecer más rápido.

La cuestión es cómo introducir y difundir las TIC en el país. La respuesta es a través de una formación sólida del uso y acceso a internet y las telecomunicaciones, ya que rompen con las barreras geográficas y difunden de manera global el conocimiento de una forma más rápida que las revoluciones tecnológicas anteriores. Castillo y Lechuga (2005, p. 62) mencionan que internet ofrece un acceso gratis a una gran cantidad de información en una gran gama de temas, además una sola conexión puede ser aprovechada por una gran cantidad de perso- nas, poniendo énfasis en la información publicitaria y noticiosa, en la que se ofrecen puestos de trabajo.

Sin embargo, Mahmoudi y Levenda (2016, p. 99) demuestran que el análisis de los impactos socioambientales de la información digital y tecnologías de la comunicación sigue siendo una empresa infructuosa y sin materializarse en el trabajo digital, por lo que sugieren un enfoque de dos partes para hacer realidad el trabajo digital: en primer lugar, pasar de la conexión de los análisis económicos y políticos de las TIC en las comunidades digitales a la coevolución y la geografía de la urbanización planetaria y el cambio tecnológico; y segundo, examinar las relaciones entre lo inmaterial, la mano de obra digital y el material industrial como sistema de producción.

En el contexto de grandes cambios tecnológicos que impactan la vida social, la urbanización y la vida laboral, muchos estudiosos han teorizado acerca de una tercera fase del capitalismo que va más allá del mercantilismo y el industrialismo, basado en el trabajo inmaterial, digital y cognitivo.

En síntesis, Mahmoudi y Levenda (2016, p. 103) muestran que las TIC son herramientas sofisticadas que requieren manejarse para iluminar las nuevas formas de pensar sobre el trabajo digital y, más ampliamente, el trabajo inmaterial. Por esto, sugieren que el trabajo inmaterial asociado a las TIC es en realidad trabajo material, de ahí que el trabajo digital $e$ inmaterial es responsable del aumento de la velocidad de circulación de capital vía internet, como un momento de la producción y un apéndice de la creciente complejidad de la industria capitalista que da pauta a la cuarta revolución industrial.

Mahmoudi y Levenda (2016, p. 105) también señalan que la materialidad del trabajo cognitivo, cultural y simbólico laboral va más allá de la ciudad e invade la vida de los residentes urbanos. La vida laboral permeada por la digitalización excreta concreto, silicio, bits, servidores y derroche de energía que produce un paisaje urbano más allá de la ciudad. A través de un examen de los centros de datos, se muestra la relación necesaria entre la urbanización de la cuarta revolución industrial y su alcance planetario en las zonas rurales desde lo digital. 
Vinculado a esto y desde un aspecto publicitario, Núñez, García y Abuín (2013) encontraron que, junto con la caída de la inversión publicitaria en México, se sucedieron cambios en la relación laboral con las agencias de publicidad, que cuestionaron la eficacia de la inversión publicitaria, y surgieron nuevas técnicas de comunicación, diferentes de la publicidad tradicional, que, a su vez, incitará un fortalecimiento de los departamentos de comunicación social de las organizaciones, entre otros cambios. En consecuencia, se tuvo la necesidad de forjar nuevas oportunidades de empleo orientadas a la digitalización económica.

Desde el punto de vista de la empresa publicitaria, surge la reestructuración de los diferentes departamentos tradicionales, por la cual se eliminaron algunos de ellos, o se ampliaron para dar cabida a las nuevas maneras de hacer comunicación publicitaria $y$ así ofrecer nuevos puestos de trabajo relacionados con la economía digital, dado que la publicidad tal y como se conocía antes (sin ser digitalizada o virtual), referida a la comunicación impersonal, hacia una audiencia masificada, empezó a quedar obsoleta.

Para Núñez, García y Abuín (2013), la transformación en el uso de mensajes publicitarios, debido a la fragmentación y diversificación de la audiencia, con múltiples canales y plataformas, así como un consumidor más crítico profesional y formado hacia la publicidad, pone en entredicho el modelo tradicional de publicidad televisiva.

En este sentido, la imagen de la marca desde lo digital se convierte en un factor de competitividad. Las ventajas competitivas están cada vez más en los activos intangibles, en los que el consumidor está más vinculado a una experiencia emocional que racional y la publicidad se reconvierte desde lo digital, ya no debe ser intrusiva, debe acompañar, ayudar al consumidor, convertirse en su marca ofreciéndole experiencias que pueden ser contadas desde las redes sociales. Desde este escenario importante es desde donde también debe cambiar buena parte del mercado laboral, sobre todo aquel relacionado con la economía digital.
El proceso de digitalización de los medios de masas tradicionales y su convergencia con las redes sociales está provocando la necesidad de profesionales capaces de crear y desarrollar contenidos digitales. En el área de la publicidad, la llegada de las redes sociales a la vida de los consumidores y su convergencia con la televisión ha provocado una verdadera revolución en el sector de la infocomunicación.

Por esto, Núñez, García y Abuín (2013) son muy claros al decir que en el área de la comunicación es necesario destacar la convergencia de los perfiles profesionales con la informática y las comunicaciones. Laboralmente hablando, el sector está demandando profesionales capaces de diseñar, programar y distribuir contenidos comunicativos y aplicaciones en el entorno digital. Es evidente que el sector publicitario y de la infocomunicación es una gran área de oportunidad para la generación de empleos; sin embargo, es necesario destacar una importante brecha entre la formación que reciben los profesionales de la publicidad y la comunicación, y las demandas del mercado laboral.

Por su parte, Kulesz (2016), desde el punto de vista de la infraestructura, se inclina por mejorar de forma decisiva el acceso a internet (en zonas rurales particularmente), e insiste en instalar puntos con wifi gratuito y ampliar la cobertura de la TV digital, mientras que para Lagunes (2016) las acciones para estar en un mercado laboral competitivo dentro de la economía digital se deben orientar hacia acciones del Gobierno de México, con el fin de impulsar los beneficios económicos del uso de internet y las TIC, así también basándose en la promoción del uso de la información pública para innovar y emprender, por lo que se institucionalizó la política de datos abiertos. Más de 3000 funcionarios de Gobierno se han capacitado en este tema y 200 dependencias están publicando más de 12000 bases de datos, que cuentan ya con más de 300000 descargas (OCDE, 2016).

De esta forma, el mercado laboral en la economía digital será fortalecido en la medida en que se proporcione el acceso a las herramientas, al conocimiento y a los medios financieros para educar, innovar e inventar usando las TIC y la fabricación 
digital, y así permitir que cualquiera pueda hacer "casi" todo y, por tanto, se puedan generar oportunidades de empleo para mejorar la vida y el sustento de todas las personas.

A medida que las tecnologías digitales permiten la producción de más bienes y servicios con menos mano de obra, también se exponen algunos trabajadores al riesgo de desempleo o de menores salarios. Pero, por otro lado, las TIC generan cambios en la organización del trabajo, que repercuten en la capacidad de políticas y programas para garantizar la inclusión en el mercado laboral, la calidad del empleo y el desarrollo de habilidades. No obstante, para capitalizar los beneficios laborales de la adopción de las TIC, los gobiernos, las empresas y las instituciones académicas tendrán que hacer frente a los nuevos retos económicos y del mercado laboral.

Este tema, que forma parte de los paneles paralelos de la Reunión Ministerial de Economía Digital, se centra en las políticas para fomentar el crecimiento y el empleo en nuevas actividades económicas habilitadas por las TIC, para acompañar a los trabajadores a lo largo de la transición a nuevos puestos de trabajo y para ayudar a garantizar la calidad del empleo en la economía digital.

\section{El mercado laboral en la economía digital de México}

De acuerdo con los datos que revela la OCDE (2016), la economía digital en México representará el 4,2\% del PBI, y según su información durante los próximos cinco años, el sector TIC conservará tasas de crecimiento promedio anual entre el 40 y el 50 $\%$, hasta tal punto que para 2025 habrá superado los USD 350000 millones. Este crecimiento colocaría a México como la segunda economía digital más grande en América Latina después de Brasil.

Según datos revelados por la Cepal (2013, p. 15), en México, la economía de internet también muestra importantes contribuciones al PIB: entre el 2 y el 2,5\% según el Boston Consulting Group y entre el 1 y el 2,2\% según McKinsey. ${ }^{1}$ De acuerdo con las

1 Las principales estimaciones de la economía digital utilizando la metodología de la demanda final son los trabajos de proyecciones, la contribución promedio para México alcanzaría el 3,5 \% hacia 2017.

Estas estimaciones en crecimiento dan respuesta a que cada vez haya mejores condiciones para el mercado laboral dentro de la economía digital, desde investigaciones tecnológicas, préstamos en línea para capital de trabajo, pagos electrónicos, plataforma de publicidad digital, comercio electrónico, marketplace de distintos servicios, inteligencia de mercado basada en big data y analyticsel, emprendimiento digital, desarrollo de infraestructura virtual, acceso a un mayor mercado de bienes y acceso a capital, hasta cambios de política pública, que van a acelerar el crecimiento y un mayor aprovechamiento de estrategias de digitalización, mejorando plataformas virtuales de pago, uso de una mayor conectividad y velocidad de internet de cada vez mejor calidad, teniendo en consecuencia un ecosistema digital orientado hacia lo laboral.

\section{Las políticas tecnológicas en México}

Los vestigios de políticas laborales con perspectiva tecnológica en México datan de hace más de tres décadas. Durante la década de los setenta en México se formularon las primeras políticas públicas enfocadas en las TIC, al diseñarse una serie de leyes de inversión cuyo propósito era desarrollar la autosuficiencia tecnológica del país. En 1970, se crea el Consejo Nacional de Ciencia y Tecnología (Conacyt) con el propósito de formular estrategias para el desarrollo científico y tecnológico de país (Cepal, 2013).

En 1981, la entonces Secretaría de Comercio y Fomento Industrial del Gobierno Mexicano (Secofi) enunció el Programa para la Promoción de la Manufactura de Sistemas Electrónicos Computacionales, cuyos objetivos eran generar una producción local de mini- y microcomputadoras, para promover su exportación y adquirir autonomía tecnológica en esta área. El programa estableció que las TIC son una herramienta fundamental para el desarrollo de una estrategia de facilitación comercial que se traduce en la reducción de costos, de tiempos

McKinsey y The Boston Consulting Group (BCG) (Cepal, 2013, p. 14). 
de gestión y de respuesta, así como en la simplificación y automatización de procesos, mayor transparencia, e incluso en la posibilidad de reorientar recursos financieros y humanos a otras áreas más sustantivas.

Este programa se entrelazó con acciones muy específicas, como el desarrollo del comercio electrónico y los medios de pago electrónicos impulsados por la Asociación Mexicana de Estándares para el Comercio Electrónico (Amece) y los bancos privados. De igual manera, la Secretaría de Hacienda y Crédito Público (SHCP) fomentó la simplificación del comercio por medio de la factura electrónica, que, a su vez, lleva incorporada la firma electrónica avanzada de los contribuyentes.

Además, Rovira y Stumpo (2013, p. 267) señalan que

el programa estableció que para incrementar la competitividad en México debería posicionarse como un eje de distribución de servicios de tecnologías de la información y de las comunicaciones y logística, lo que implicó una agresiva estrategia de formación de recursos humanos para dotar al sector de TIC con el capital humano necesario.

Esto tiene implicaciones tanto en el mercado laboral como en las nuevas generaciones de profesionales que incursionarian en el mundo de la economía digital y el mundo de los negocios con habilidades para implementar las TIC en sus modelos de negocio, ampliando la adopción de estas en el sector empresarial.

Este programa, que forma parte de la estrategia de desarrollo del mercado interno establecida en el Prosoft 2.0, fue diseñado según el Plan Nacional de Desarrollo 2007-2012 con el objetivo de crear las condiciones para que México cuente con un sector de servicios basados en las TIC competitivo internacionalmente y asegurar su crecimiento a largo plazo, así como promover el uso y un mejor aprovechamiento de estas tecnologías en el mercado laboral.
Según la información que revelan Rovira y Stumpo (2013, p. 274), más de la mitad de las estrategias del Prosoft están relacionadas con el fomento del uso y de la adopción de las TIC en el sector productivo y laboral, entre ellas, la educación y formación de personal competente en el desarrollo de software, en la cantidad y calidad convenientes. Con esto, se busca desarrollar competencias en los ingenieros y técnicos que se encuentran en el mercado laboral, además de influir en la adecuación de los planes de estudio para que sean acordes con las necesidades del mercado laboral.

Para alinear la oferta y la demanda, la Secretaría de Economía diseñó un conjunto de programas cuyo propósito fue incrementar las capacidades del mercado laboral y reducir la brecha existente entre la oferta actual de recursos humanos en el área de las TIC y la demanda del sector productivo, incluso, por supuesto, la demanda de la misma industria de las TIC.

Estos programas enmarcados en el proyecto Primero México se crearon para la certificación con reconocimiento internacional; Proyecto BID (modelo paracurricular) para el desarrollo de perfiles profesionales de Ingeniero de Software, Arquitecto de Software, Administrador de Proyectos y Procesos de Software y Emprendedor de Negocios de Software. Los programas se trabajaron conjuntamente con la Secretaría de Educación Pública (SEP) a través de Impúlsate con Inglés (capacitación del sector productivo en inglés), de competencias profesionales del nivel medio superior (escuelas preparatorias) y de normalización de competencias profesionales por medio del Consejo Nacional de Normalización y Certificación de Competencias Laborales (Conocer).

\section{La Agenda Digital Nacional}

La Agenda Digital Nacional (ADN) se origina en 2009 como una iniciativa del Gobierno de México para el desarrollo de la sociedad de la información. En enero de 2009, la Secretaría de la Función Pública (SFP) elaboró la Agenda de Gobierno Digital (AGD), que fue publicada en el Diario Oficial de la Federación con el propósito de establecer las 
estrategias para el desarrollo del gobierno digital, en apoyo al Programa de Mejora de la Gestión (PMG), y de otorgar mejores servicios, facilitar el acceso a la información, la rendición de cuentas y la transparencia, y fortalecer la participación ciudadana.

La ADN constituyó un documento inédito, que trazó el camino en materia de TIC a toda la Administración pública federal para el control y manejo de la economía con el objeto de dejar de lado los esfuerzos aislados en cada dependencia oficial. En la actualidad, la agenda digital del país se ha reformulado en concentrarse en abrir oportunidades laborales en la era digital, a fin de extender las áreas de oportunidad de formación y empleabilidad en la economía digital, lo que significa competir decididamente por el talento del capital humano y crear las estructuras adecuadas para ello.

Así, en los pronunciamientos del Gobierno federal, desde 2015 a la fecha, se desarrollan dos iniciativas: por un lado, se promueve la empleabilidad en oficios a través de cursos de capacitación general o especializada en competencias en las TIC. Por otro, se avanza en la definición y actualización de los perfiles técnicos y académicos requeridos en el mercado laboral.

En este sentido, abrir más y mejores oportunidades laborales significa una mayor enseñanza en las TIC, dado que son un instrumento fundamental para desarrollar las oportunidades de formación y trabajo de todas las personas a través de la economía digital tanto para la oferta como para la demanda laboral. Por ello, en México, se canalizan sinergias de distintos programas públicos orientados a la inserción y reinserción laboral mediante el uso de las TIC, así como algunas herramientas tecnológicas para la búsqueda de empleo. Además, hay módulos de formación de las TIC en oficios para impulsar el acceso y la permanencia en el mercado laboral de mujeres y jóvenes en condiciones de vulnerabilidad, con énfasis en capacidades diferentes y necesidades especiales.

No obstante, para finalizar, se menciona que la adecuación de la demanda y oferta del volumen y el perfil de los profesionales de las TIC es un asunto pendiente en la ADN. Para resolverlo, la Secretaría del Trabajo y la Secretaría de Educación Pública aplican instrumentos relacionados con el sistema de definición de competencias laborales y cualificaciones en las TIC, como el establecimiento de perfiles de carácter técnico-profesional y la certificación de competencias laborales de acuerdo con estándares internacionales.

\section{Conclusiones}

Como se puede mostrar, el mercado laboral y sus estructuras ante las TIC se encuentran inmersos en condiciones tecnológicas avanzadas que implican riesgos pero también oportunidades para trabajadores, y así les permite ser más productivos a través de la interconectividad.

Se observa que en la economía digital el mercado laboral podría ser un eslabón de competitividad laboral dentro de la cadena de valor para los próximos diez años, pese a que los organismos internacionales advierten sobre la crisis de los mercados laborales a causa del elevado desempleo. No obstante, se reconoce que, como consecuencia del uso extensivo de las TIC, se ha generado una pérdida masiva de empleo, debido a que se automatizan las tareas rutinarias del trabajo y se presenta la necesidad de una mayor capacitación en las TIC para mitigar el riesgo del atraso en las actividades cognoscitivas y de experiencia laboral, que se consideraban no automatizables.

Desde el punto de vista teórico y empírico, se presentan los argumentos más aceptados y respaldados por Acemoglu y Restrepo (2016, 2017), Arntz, Gregory y Zierahn (2016), BM (2016), Cepal (2013) y Frey y Osborne (2017), al referirse a algunas de las expresiones más pesimistas que indican que cerca de la mitad de los empleos en países como los Estados Unidos y tres cuartas partes de los empleos en países como Brasil y México están en riesgo de ser sustituidos por máquinas dentro de los próximos veinte años, lo que abriría paso a la robotización laboral.

Para evitar ambigüedades, a lo largo del trabajo se dan argumentos teóricos y empíricos que se 
orientan al logro del objetivo de este, que es propiamente analizar las amenazas y oportunidades que desde la economía digital tiene el mercado laboral, poniendo énfasis en la perspectiva de creación de empleos o extinción de estos por el desplazamiento de mano de obra ante la fuerza de las TIC. Se explica el caso de la política pública mexicana y la articulación de la agenda digital con el mercado laboral, haciendo hincapié en los efectos de las TIC sobre los trabajadores y el empleo citado en los diferentes programas, como el Programa de Mejora de la Gestión Laboral y el Prosoft.

Ahora bien, se desestimaría cualquier ambigüedad ante una posición teórica "optimista" acerca de que la introducción de las TIC genera nuevos trabajos y nuevas oportunidades de empleo, que, más que una aseveración "optimista", merece reconocer que sí los genera; sin embargo, habría que considerar una afirmación "menos positiva" al observar que las TIC también reemplazan mano de obra, destruyen puestos de trabajo tradicionales, que de no orientarse al uso de las TIC y a los procesos digitales, estos puestos de trabajo tenderían a desaparecer de la economía por estacionarse en un estadio antiguo y primitivo.

Estos programas ofrecen respuestas acerca de la automatización de los procesos en las actividades de trabajo que muestran diversos efectos en el mercado laboral. Mientras que las TIC pueden desplazar a trabajadores que desempeñan determinados trabajos de ciertos sectores de la economía, esto también implica la disminución de sus oportunidades de empleo y sus salarios. Otros sectores y ocupaciones podrían extenderse y absorber la mano de obra que se libera por las actividades que realizan las máquinas o través de las TIC, e incluso, es posible que los dividendos en productividad originados por tecnologías disruptivas amplíen las posibilidades de empleo en los sectores económicos en los que se extienden las TIC.

Según esto, se observa que existe un gran interés en las tecnologías cambiantes como herramientas para el trabajo creativo; sin embargo, gran parte de las aportaciones teóricas inciden en el posicionamiento del trabajo digital como parte del mercado laboral que lo caracteriza como el eslabón de competitividad en la economía digital.

La capacidad de trabajadores y empleadores de adaptarse a las tecnologías cambiantes es identificada como la resiliencia laboral digital que impacta la estructura del mercado laboral, entendiendo que la resiliencia laboral digital es la capacidad de adaptación al cambio tecnológico que tienen empleadores y trabajadores, tomando de manera positiva las dificultades y disponiéndose a capacitarse para superar los obstáculos y los riesgos que las TIC les imponen y, a su vez, obtener el máximo beneficio en toda la estructura del mercado laboral.

Finalmente, se considera que el uso de las TIC, así como ofrece amenazas al mercado laboral, también brinda oportunidades a los trabajadores, siempre que se adapten a los cambios que el ecosistema digital establece. De lo contrario, si los empleadores y los trabajadores no se adaptan al uso de las TIC, corren un alto riesgo de perder el eslabón de competitividad laboral y empresarial y, con ello, se caería en procesos arcaicos y rudimentarios. Perder competitividad empresarial (para el caso de las organizaciones) y competitividad laboral (para el caso de los trabajadores) implicaría desaparecer de la economía digital.

\section{Referencias}

Acemoglu, D. y Restrepo, P. (2016). The race between machine and man: Implications of technology for growth, factor shares and employment. NBER Working Paper, 22252. Recuperado de http://www.nber.org/papers/w22252

Acemoglu, D. y Restrepo, P. (2017). Robots and jobs: Evidence from US labor markets. NBER Working Paper, w23285. Recuperado de https://papers.ssrn.com/sol3/papers. cfm?abstract_id $=2941263$

Arntz, M., Gregory, T. y Zierahn, U. (2016). The risk of automation for jobs in OECD countries. OECD Social, Employment and Migration Working Papers, 189. Recuperado de https:// www.oecd-ilibrary.org/docserver/5jlz9h56dvq7-en.pdf?exp ires $=1536406651 \&$ id $=$ id\&accname $=$ guest $\&$ checksum $=59$ C01F42673F586A4E8CA845DE1B0713

Banco Mundial (2016). Informe sobre el desarrollo mundial 2016. Dividendos digitales: panorama general. Washington D. C.: Banco Mundial. Recuperado de http://documents.worldbank. org/curated/en/658821468186546535/pdf/102724WDR-WDR2016Overview-SPANISH-WebResBox-394840BOUO-9.pdf 
Campos Freire, F. y Alonso Ramos, N. (2015). Las herramientas digitales sociales en línea para la autopromoción laboral: hacia un estado de la cuestión. Revista Latina de Comunicación Social, 70, 288-299.

Castillo Soto, M. y Lechuga Montenegro, J. (2005). Las tecnologías de la información y la nueva economía. En J. Lechuga Montenegro, Reflexiones acerca de la era de la información (new economics). México: Universidad Autónoma Metropolitana.

Centro de Estudios de las Finanzas Pública (2017, enero 18). Aspectos relevantes de la competitividad del sector industrial. Recuperado de http://www.cefp.gob.mx/publicaciones/ documento/2017/eecefp0012017.pdf

Comisión Económica para América Latina y el Caribe (2013). Economía digital para el cambio estructural y la igualdad. Santiago de Chile: Comisión Económica para América Latina y el Caribe.

Dahms, T. (2010). Resilience and risk management. The Australian Journal of Emergency Management, 25(2), 21-26.

Diodato, D. y Weterings, A. B. (2015). The resilience of regional labour markets to economic shocks: Exploring the role of interactions among firms and workers. Journal of Economic Geography, 15(4), 723-742.

Duffy, B. E. (2016). The romance of work: Gender and aspirational labour in the digital culture industries. International Journal of Cultural Studies, 19(4), 441-457.

Frey, C. B. y Osborne, M. A. (2017). The future of employment: How susceptible are jobs to computerisation? Technological Forecasting and Social Change, 114, 254-280. Recuperado de https://www.sciencedirect.com/science/article/pii/ S0040162516302244

Goos, M., Konings, J. y Vandeweyer, M. (2015). Employment growth in Europe: The roles of innovation, local job multipliers and institutions. Recuperado de https://papers.ssrn. com/sol3/papers.cfm?abstract_id=2671765

Kulesz, O. (2016). Intergovernmental committee for the protection and promotion of the diversity of cultural expressions. París: Unesco. Recuperado de https://en.unesco.org/creativity/ sites/creativity/files/sessions/10igc_inf4_the_impact_of_digital_technologies_octavio_kulesz_en.pdf
Lagunes, A. (2016, junio 23). Las nuevas fronteras de la economía digital. El Financiero. Recuperado de http://www.elfinanciero. com.mx/opinion/alejandra-lagunes/las-nuevas-fronteras-dela-economia-digital

López León, M. E. y Saladrigas Medina, H. (2016). Las TIC: un nuevo escenario para el desarrollo local de las comunidades. Opción, 32(10), 71-94.

Mahmoudi, D. y Levenda, A. M. (2016). Beyond the screen: Uneven geographies, digital labour, and the city of cognitive-cultural capitalism. Journal for a Global Sustainable Information Society, 14(1), 99-120.

Moretti, E. (2010). Local multipliers. American Economic Review, 100(2), 373-77.

Nottebohm, O., Manyika, J., Bughin, J., Chui, M. y Syed, A. (2012). Online and upcoming: The internet's impact on aspiring countries. McKinsey \& Company.

Núñez, P., García, A. y Abuín, N. (2013). Profesionales digitales en publicidad y comunicación: una aproximación a las necesidades del mercado laboral. CIC: Cuadernos de Información y Comunicación, 18, 177-187.

Organización para la Cooperación y el Desarrollo Económicos (2016). Perspectivas de la OCDE sobre la economía digital 2015. México: Microsoft México.

Rovira, S. y Stumpo, G. (comps.) (2013). Entre mitos y realidades: TIC, políticas públicas y desarrollo productivo en América Latina. Santiago de Chile: Comisión Económica para América Latina y el Caribe.

Ruberg, B. (2016). Doing it for free: Digital labour and the fantasy of amateur online pornography. Porn Studies, 3(2), 147-159.

Tabarés, R. (2016, junio 26). ¿La economía digital promueve la desigualdad? [Entrada blog]. Recuperado de https://ssociologos.com/2016/06/26/la-economia-digital-promueve-ladesigualdad/

Weller, J. (2017). Las transformaciones tecnológicas y su impacto en los mercados laborales. Santiago de Chile: Comisión Económica para América Latina y el Caribe. 